\title{
Low temperature magnetization and resistivity measurements in Co based soft magnetic microwires
}

\author{
J. Llumá ${ }^{\mathrm{b}}$, M. Vázquez ${ }^{\mathrm{a}, *}$, J.M. Hernandez ${ }^{\mathrm{b}}$, J.M. Ruiz ${ }^{\mathrm{b}}$, J.M. García-Beneytez ${ }^{\mathrm{a}}$, \\ A. Zhukov', F.J. Castaño ${ }^{a}$, X.X. Zhang ${ }^{b}$, J. Tejada ${ }^{b}$ \\ "Instituto Magnetismo Aplicado and Instiluto Ciencia Materiales. P.O. Box 155, E-28230 Madrid, Spain \\ 'Departmento Física Fonamental. Universitat de Barcelona. E-08028 Barcelona, Spain
}

\begin{abstract}
Magnetization and resistivity measurements, embracing a temperature range $2-300 \mathrm{~K}$, have been performed in glass-coated $\mathrm{Co}_{68} \mathrm{Mn}_{7} \mathrm{Si}_{10} \mathrm{~B}_{15}$ amorphous microwires. The relation between both types of measurements is analysed. Similar trends of the temperature dependence of the domain structure, magnetization and resistivity are observed. An increase of resistance is found for the saturated state. This increase of the resistivity is analysed in terms of the domain structure and the presence of domain walls. (C) 1999 Elsevier Science B.V. All rights reserved.
\end{abstract}

Keywords: Amorphous materials; Magnetization process; Resistivity; Magnetoresistance

Considerable interest has been devoted recently to the effect of the magnetic domain walls on the electron transport properties of amorphous metallic systems. In this way, substantial theoretical effort has been reported concerning these effects [1-3], although a clear thcoretical picture still remains controversial. Moreover, the possibility of macroscopic quantum effects connected with the depinning of domain walls and magnetization reversal has triggered an even more exciting research topic $[4,5]$. Several reports have recently appeared on such effects although it must be emphasized that only few of them include experimental results on the magnetization and domain structure when studying their influence on the electrical resistivity [6,7]. In some cases, the interpretation of magnetization process is analyzed indirectly through the field dependence of resistivity, which is typically easier to be checked [8].

Glass-coated amorphous ferromagnetic microwires are formed of a metallic nucleus protected by an insulating Pyrex-like coating. This type of magnetic amorphous

\footnotetext{
*Corresponding author. Tel.: + 34-91-6301724; fax: + 3491-6301625; e-mail: vazquezv@fenix.ima.csic.es.
}

microwires exhibit outstanding magnetic properties arising mainly form their fabrication procedure [9]. For example, perfectly square shaped or quasi-non-hysteric hysteresis loops are obtained depending on the magnetostriction constant which finally determines the domain structure and the overall magnetization process.

$\mathrm{Co}_{68} \mathrm{Mn}_{7} \mathrm{Si}_{10} \mathrm{~B}_{15}$ glass-coated microwires were fabricated using the Taylor-Ulitovsky technique. The diameter of the metallic nucleus was about $5 \mu \mathrm{m}$ and the total thickness, including the Pyrex coating, reached $21 \mu \mathrm{m}$. The microwires investigated were thermally treated at $150^{\circ} \mathrm{C}$ during $1 \mathrm{~h}$ in the presence of an external axial magnetic field of $180 \mathrm{Oe}$.

The hysteresis loops were measured at room temperature using an induction technique at $80 \mathrm{~Hz}$. These measurements show that the magnetization process mainly takes place due to a large Barkhausen jump caused by the movement of a reduced number of domain walls. Perfect square loops are only reached when the magnetization reversal takes place by the displacement of a single domain wall. This process is widely analysed in soft magnetic wires elsewhere [10].

Low-temperature magnetic measurements were performed on $1 \mathrm{~cm}$ long $\mathrm{Co}_{68} \mathrm{Mn}_{7} \mathrm{Si}_{10} \mathrm{~B}_{15}$ microwires using 
a SQUID magnetometer. Fig. 1 shows the magnetization curves for the temperature range $2-300 \mathrm{~K}$. Relevant information on the magnetization process and domain structure can be derived from the analysis of these curves.

Fig. 2 shows the temperalure dependence of both the anisotropy field and the remanence deduced from these measurements. $\Lambda$ change of the magnetization process can be inferred: at high temperatures displacements of axial domain walls dominate, while at low temperatures magnetization rotation is responsible for the process. The domain structure, determined by the stress distribution and magnetostriction [9], consists of an inner corc and an outer shell, with axial and circumferential easy magnetizations directions respectively. In the absence of magnetic fields, the volume fraction occupied by the core increases with temperature, reaching its maximum value when the remanence approaches the saturation magnetization $(T \sim 100 \mathrm{~K})$. This thermal dependence of the domain structure at the remanent state can be regarded to the presence of internal stresses arising from the different expansion coefficients of the metallic nucleus and glass coating. These results show an almost linear behavior of the anisotropy field with temperature (see Fig. 2).

Resistivity measurements have been performed with the four points method on the same $6 \mathrm{~cm}$ long microwire, using two equipments: a gas flow cryostat measuring resistance at $0.1 \mathrm{~Hz}$ between 1.8 and $300 \mathrm{~K}$, and a dilution refrigerator measuring at $12.5 \mathrm{~Hz}$ in the $0.04-2 \mathrm{~K}$ temperature rangc. In order to obtain a good resolution with low power dissipation, the resistance deviation from external reference resistor is measured instead of the total resistance when acquiring $R(H)$ curves below $2 \mathrm{~K}$. The accuracy in resistance measurement is better than $0.1 \Omega$ and in the deviation measurements, better than $10 \mathrm{~m} \Omega$.

The temperature evolution of the resistivity, $R_{0}$ is shown in Fig. 3 (note the semi-logarithmic scale). There are two well defined regions: for temperatures below

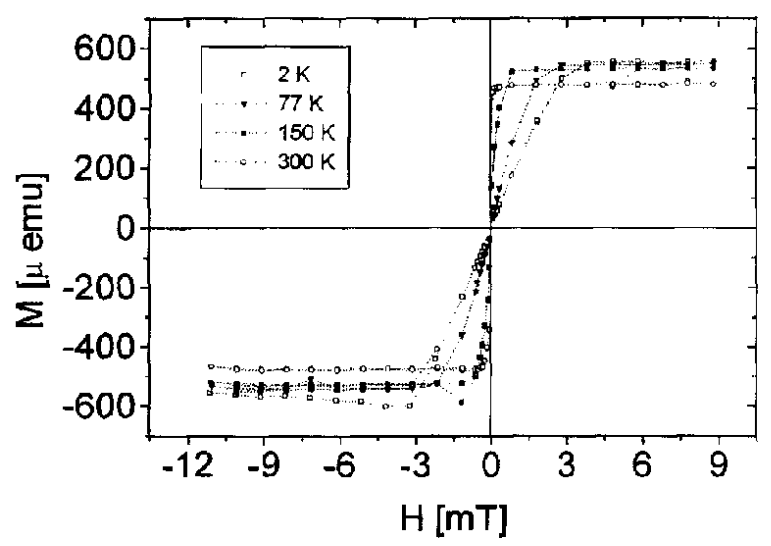

Fig. 1. Temperature dependence of the magnetization curves for the CoMnSiB glass-coated microwire.

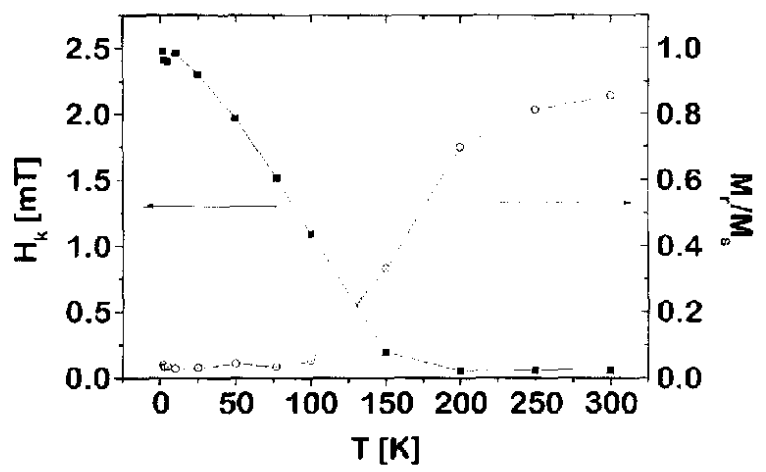

Fig. 2. Temperature evolution of the anisotropy field. $I_{\mathrm{K}}$, and reduced remanence, $M_{\mathrm{R}} / M_{\mathrm{s}}$ deduced from results given in Fig. 1 .

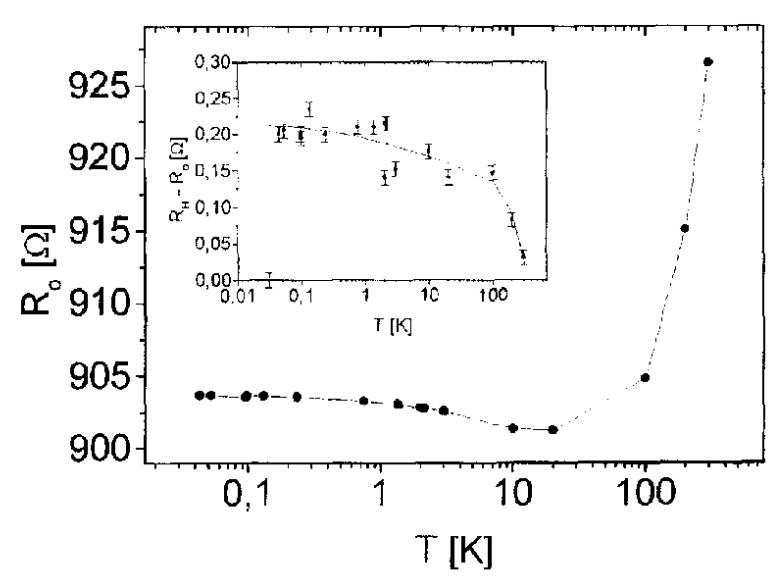

Fig. 3. Temperature dependence of the resistance, $R_{0,}$ and of its increase, $R_{\mathrm{H}} \quad R_{0}$, when an axial field of $80 \mathrm{Oe}$ is applied.

$100 \mathrm{~K}$, the resistivity slightly decreases with increasing temperature, while an almost linear increasing behavior is found above $100 \mathrm{~K}$. The effect of an external axial magnetic field of 80 Oe has also been measured. This field is large enough to reach magnetic saturation (see Fig. 1) within the whole temperature range. The inset in Fig. 3 shows the difference in the resistivity between the saturation and the remanent states. The resistivity increases when the field is applied over the entire temperature range, reaching a maximum relative change of $0.02 \%$.

In the low temperature region, the decrease of the resistivity with the temperature can be interpreted considering quantum contributions to the electron scattering [11], while at higher temperatures, the resistivity increases almost linearly as expected for conventional metals.

The above presented experimental results reveal that both the thermal evolution of the magnetic (Fig. 2) and transport (Fig. 3) properties exhibit a change in their behavior for temperatures around $100 \mathrm{~K}$. suggesting 
a correlation between them. At low temperatures, the magnetization rotates from the circumferential easy axes to the axial direction. This rotation is carried over domains which are separated by circular walls. In this case, the increase of the resistivity observed when a magnetic field is applied is relatively large. At higher temperatures, the axial magnetization process takes place by displacements of axial walls, and the increase in the resistivity $\left(R_{\mathrm{H}}-R_{0}\right)$ is smaller as compared with the previous case. This can be ascribed to the decrease of the volume fraction which contributes to the magnetoresistance owing to its easy transverse magnetization direction. In both temperature regimes, the resistivity increases when a saturating field eliminates domain walls. As pointed out recently [2], the presence of nucleated walls may lcad to a reduction of the resistance due to the decoherence of conduction electrons. Additionally, another contribution can be found at low temperatures associated with the increase of the anisotropy field and the consequent reduction of the domain wall thickness.

In summary, in the presently studied microwires, a simultaneous change in the magnetic and transport properties has been found experimentally at low temperatures. When the magnetization process is dominated by domain wall motion (room temperature), the total resistivity increases when temperature increases and there is no significant change in its value when a magnetic field is applied. When domain rotation takes place (low temperature), the total resistivity is almost constant with $T$ and the measured magnetoresistance becomes larger. These results suggest that the presence of domain walls play a significant role in the scattering of conduction electrons.

\section{References}

[1] P.M. Levy, S. Zhang, Phys. Rev. Lett. 79 (1997) 5110.

[2] G. Tatara, H. Fukuyama, Phys. Rev. Lett. 78 (1997) 3773.

[3] J.B.A.N. Van Hoof, K.M. Schep, P.J. Kelly, G.E.W. Bauer, J. Magn. Magn. Mater. 177-181 (1998) 188.

[4] K. Hong. N. Giordano, J. Phys.: Condens. Matter 8 (1996) L301.

[5] E.M. Chudnosky, J. Tejada, Macroscopic Quantum Tunneling of the Magnetic Moment, Cambridge University Press, Cambridge, 1998.

[6] J.F. Gregg, W. Allen, K. Ounajdela, M. Viret, M. Hehn, S.M. Thompson, J.M.D. Coey, Phys. Rev. Lett. 77 (1996) 1580.

[7] C. Shearwood, S.J. Blundell, M.J. Baird, J.A.C. Bland, M. Gester, H. Ahmed, H.P. Huges, J. Appl. Phys. 75 (1994) 5249.

[8] K. Hong, N. Giordano, Phys. Rev. B 51 (1995) 9855.

[9] M. Vázquez, A.P. Zhukov, J. Magn. Magn. Mater 160 (1996) 223.

[10] M. Vázquez, D.-X. Chen, IEEE Trans. Magn. 31 (1994) 1229.

[11] I.M. Lifshits, Quantum Theory of Solids, MIR, Moscow, 1982. 